Relations industrielles

Industrial Relations

\title{
Juridiction des tribunaux du travail
}

\section{Gérard Picard}

Volume 16, numéro 3, juillet 1961

URI : https://id.erudit.org/iderudit/1021777ar

DOI : https://doi.org/10.7202/1021777ar

Aller au sommaire du numéro

Éditeur(s)

Département des relations industrielles de l'Université Laval

ISSN

0034-379X (imprimé)

1703-8138 (numérique)

Découvrir la revue

Citer ce document

Picard, G. (1961). Juridiction des tribunaux du travail. Relations industrielles / Industrial Relations, 16(3), 354-356. https://doi.org/10.7202/1021777ar

Tous droits réservés @ Département des relations industrielles de l’Université Laval, 1961
Ce document est protégé par la loi sur le droit d'auteur. L’utilisation des services d'Érudit (y compris la reproduction) est assujettie à sa politique d'utilisation que vous pouvez consulter en ligne.

https://apropos.erudit.org/fr/usagers/politique-dutilisation/ 
également des tribunaux de travail statuant en appel et sur recours en cassation.

Tantôt on a confié à ces tribunaux une compétence uniquement pour les conflits collectifs du travail qui mettent en cause des associations professionnelles et portent sur l'interprétation d'une convention collective, la licéité d'une grève ou lock-out, etc., c'est en principe le système adopté par les pays scandinaves. Allant plus loin. les lois d'autres pays soumettent aux tribunaux de travail tout conflit, individuel ou collectif, relatif à un rapport de travail ou né à l'occasion du travail.

D'autre part il faut rappeler que le développement de la législation sociale et des conventions collectives a engendré une nouvelle branche importante du droit administratif. Les décisions prises par les inspecteurs du travail, les commissions des relations industrielles et d'autres services administratifs en application des règles de ce nouveau droit social peuvent, selon le cas, faire l'objet d'un recours contenticux. Lorsqu'il existe des tribunaux administratifs d'appel et de cassation, ces recours peuvent porter non seulement sur la forme mais aussi sur le fond de la décision entreprise. Ailleurs - et tel est le cas du Canada - le recours contre une décision administrative est de la compétence des tribunaux de droit commun. Mais ceux-ci ne peuvent annuler la décision administrative que pour vices de forme et n'ont pas le droit d'en examiner le bien-fondé. Or, il est intéressant de noter que les pays possédant des tribunaux du travail leur ont parfois confié compétence pour connaître l'appel contre des décisions des services chargés de l'application du droit administratif du travail. Ces appels qui permettent un nouveau débat sur le fond de la question litigieuse, sont, toutefois, soumis à une procédure différente de celle régissant des conflits de droit civil.

L'expérience faite à l'étranger avec les tribunaux de travail est concluante. Leur procédure est rapide et expéditive. Le nombre des litiges se terminant par un règlement à l'amiable est assez considérable. On a également constaté que les assesseurs représentant respectivement les travailleurs et les employeurs s'habituent très vite à juger en droit, sans se laisser influencer par leurs préjugés professionnels.

Enfin les tribunaux de travail ont contribué au développement du droit privé en donnant au droit privé et à la législation ouvrière une interprétation qui répond aux besoins et particularités du monde du travail.

\section{Juridiction des tribunaux du travail}

\section{Gérard Picard}

La juridiction des tribunaux du travail devrait, à mon avis, embrasser toute la Province de Québec et s'étendre (à défaut d'un Code provincial du Travail) aux lois du travail, à la sécurité sociale et à l'assistance sociale.

Les tribunaux du travail ayant ainsi une juridiction civile et une juridiction pénale devraient se situer, dans la hiérarchie judiciaire, entre l'arbitrage et la Cour Supérieure.

L'existence de tribunaux du travail ne devrait pas, pour éviter un conflit majeur au point de départ, affecter le régime de négociations collectives, ni remplacer la conciliation volontaire, l'arbitrage volontaire ou la médiation volontaire, et encore moins viser à interdire le recours à la grève, ce droit relevant du Code pénal canadien. 
Nos tribunaux civils ordinaires entendent et jugent, le plus souvent, des causes où sont aux prises des citoyens, individuellement. Il ne faut pas s'en surprendre. Les parties à ces litiges s'appuient, pour faire reconnaître leurs droits, sur l'une ou l'autre des dispositions du Code civil de la Province de Québec. Ce Code civil régit les rapports juridiques des citoyens entre eux. Il ne régit pas les rapports des collectivités entre elles. Notre Code civil ne connaît pas les organisations syndicales de travailleurs, ni les associations patronales, ni les conventions collectives de travail, ni la sécurité sociale à l'exception de la théorie du risque professionnel (la théorie de la faute ayant été écartée) en matière d'accidents du travail.

De plus, la procédure à suivre (Code de Procédure civile) pour déclencher le mécanisme de l'appareil judiciaire de nos tribunaux civils est une procédure trop lente, trop formaliste et trop dispendieuse en regard des problèmes du travail et de la sécurité sociale.

C'est probablement ce qui explique que, depuis une vingtaine d'années surtout, les organismes consultatifs, administratifs et quasi-judiciaires se sont multipliés dans divers domaines de l'activité économique et sociale, et plus particulièrement dans le domaine des lois du travail et de la sécurité sociale (Exemples: Conseil Supérieur du Travail, Commission des Accidents du Travail, Commission du Salaire Minimum, Commission de Relations ouvrières, etc.)

Pour rendre la procédure encore plus expéditive devant ces divers organismes, le législateur a tenté, pas toujours avec succès, d'interdire le recours aux brefs de prérogatives (Qua Warranto, Mandamus, Certiorari, bref de prohibition et injonction), de même qu'il a tenté de les soustraire à la surveillance et au contrôle de la Cour Supérieure (Art. 50 du Code de Procédure civile).

Comment remédier à cette situation d'une manière rationnelle et efficacé En ajoutant, dans notre système judiciaire, une juridiction du travail, c'est-à-dire des tribunaux du travail et une Magistrature du Travail, et en posant les limites de cette juridiction.

Il devrait y avoir, à mon avis, un tribunal du travail et un greffe du travail dans chaque district judiciaire. Chaque tribunal du travail devrait comprendre un juge (impartial et compétent), un assesseur syndical et un assesseur patronal. Ces deux derniers avec voix délibérative seulement. Le jugement devrait être rendu par le juge seul.

En plus de la juridiction générale suggérée au début de ces notes: juridiction civile (réclamations) et juridiction pénale (infractions), un tribunal du travail devrait avoir une juridiction d'appel permettant de se pourvoir contre le défaut ou l'excès de juridiction d'un arbitre ou d'un conseil d'arbitrage (pas un conseil de conciliation), et contre le déni de justice de la part d'un arbitre ou d'un conseil d’arbitrage.

De plus, pour que la situation soit claire, lorsqu'un tribunal du travail siège en première instance et qu'il s'agit d'une réclamation assez élevée (montant à déterminer), ce tribunal du travail devrait rester soumis au droit de surveillance et de contrôle de la Cour Supérieure, vu que cette Cour est le coeur du système judiciaire dans la Province de Québec. Même raisonnement, dans le cas d'un 
tribunal du travail siégeant en première instance, s'il s'agit de se pourvoir contre le défaut ou l'excès de juridiction ou contre un déni de justice.

Enfin, sur accord des parties, un tribunal du travail devrait pouvoir juger des conflits de droits pendant la durée d'une convention collective, de même, par exemple, des cas de congédiements qui sont actuellement référés à la Commission de Relations ouvrières. Ce qui ne signifie pas une approbotion de la juridiction qui resterait à la CRO selon la loi actuelle.

\section{La composition et le système de procédure des tribunaux du travail}

\section{André Desgagné}

\section{LE PROBLÈME}

Après avoir fourni les motifs qui justifient la mise sur pied de tribunaux spécialisés en matière de travail, il convient de s'interroger sur l'organisation de tels tribunaux. A cet égard, il n'est pas suffisant de décrire le champ (compétence matérielle) et le «détroit» (compétence personnelle) de tribunaux projetés, il faut encore savoir quelle en sera la composition, puis quel système de procédures y sera en vigueur. En d'autres termes, il faut en outre se demander qui jugera et suivant quelles formes on jugera.

\section{A. Composition des tribunaux du travaII}

La question de la composition des tribunaux du travail, comme celle de tout tribunal, soulève tout d'abord le problème de savoir si les juges seront de carrière ou d'occasion et, en tout cas, le problème des conditions d'éligibilité à la charge de juge; elle soulève en second lieu le problème de la collégialité ou du juge unique.

Nous répondrons à ces deux séries de problèmes en montrant les inconvénients et les avantages de chacune des alternatives et conclurons en faveur d'une formule tirant avantage de chacune d'elles.

\section{B. Le Système de PROCÉdures des tribunaux DU TRAVAIL}

Après avoir rappelé qu'il existe deux grands types de système procédural - le type libéral et le type inquisitoire - spécialement caractérisés, le premier, par la prépondérance du juge, le second, par celle des parties, nous montrerons comment se pose cette option concrètement en reprenant chacune des phases d'une instance: phase initiale (institution de l'instance), phase intermédiaire (instruction), phase finale (jugement).

1) Phase initiale: l'option est la suivante: l'initiative de mettre en branle les rouages des tribunaux du travail peut-elle être laissée aux parties impliquées dans le rapport de travail ou être attribuée à un organisme d'Etat ou être partagée par l'un et l'autre?

2) Phase intermédiaire: l'option principale se présente en matière de preuve. La preuve doit-elle être laissée à l'entière discrétion des parties ou non?

3) Phase finale: ici, il y a deux options importantes à faire: les jugements doivent-ils être motivés ou non; les jugements sont-ils susceptibles d'appel ou non? 